\title{
Simulations and background estimates for the DAMIC-M experiment
}

\author{
Claudia De Dominicis ${ }^{a, *}$ and Mariangela Settimo ${ }^{a}$ \\ ${ }^{a}$ SUBATECH, IMT Atlantique, Université de Nantes, CNRS-IN2P3, \\ 4 rue Alfred Kastler, F-44300, Nantes, France \\ E-mail: dedomini@subatech.in2p3.fr, settimo@subatech.in2p3.fr
}

DAMIC-M (Dark Matter in CCDs at Modane) is a near-future experiment aiming to search for low-mass dark matter particles through their interactions with silicon atoms in the bulk of chargecoupled devices (CCDs). This technique was pioneered by the DAMIC experiment at SNOLAB. Its successor DAMIC-M will have a 25 times larger detector mass and will employ a novel CCD technology (skipper amplifiers) which allows to achieve a readout noise of 0.07 electrons. With these novelties, DAMIC-M will reach unprecedented sensitivities to dark matter candidates of the so-called hidden sector. A challenging requirement is the control of the radiogenic background at the level of a fraction of events per $\mathrm{keV}$ per kg-day of target exposure. Accurate Geant 4 simulations are being employed to optimise the detector design and drive the material selection and handling. This proceedings provides a comprehensive overview of the explored detector designs, the estimated background, and the strategies for its mitigation.

$37^{\text {th }}$ International Cosmic Ray Conference (ICRC 2021)

July 12 th - 23rd, 2021

Online - Berlin, Germany

\footnotetext{
${ }^{*}$ Presenter
} 


\section{Introduction}

Nowadays the $\Lambda \mathrm{CDM}$ standard model of cosmology indicates that only $5 \%$ of the total energymatter density of the Universe is made of ordinary matter, while about $27 \%$ consists of unassessable matter known as Dark Matter (DM) [1]. Many DM models and candidates have been proposed. In one of the most popular models, the dark matter could be in the form of a weakly interacting massive particle (WIMP), with a mass in the few GeV-TeV range. This particle may scatter off nuclei which, by recoiling, would produce a detectable signal. However, no WIMP signals have been detected so far and alternative candidates are becoming more and more popular. Among them there are the so-called hidden sector particles [2].

The DAMIC-M (Dark Matter In CCD at Modane) experiment searches for DM particle interactions in the silicon bulk of charge-coupled devices (CCDs). With its sub-electron resolution and low background, it will be leading the search of $\mathrm{MeV}$-scale DM candidates in the hidden sector and eV-scale hidden photon. The CCDs are light sensors commonly used in digital cameras and telescopes for astronomical applications. Their applicability in the search for DM was successfully demonstrated by DAMIC, the predecessor of DAMIC-M located at SNOLAB. DAMIC-M will be installed at the Laboratoire Souterrain de Modane (LSM) in France and will increase by 25 times the mass of its detector. It will employ the so-called skipper CCDs to achieve sub-electron resolution on the charge measurements [3]. Due to these features and to a low level of dark current, DAMIC-M will be extremely sensitive to very feeble ionization signals down to few $\mathrm{eV}$, which are expected from DM interaction with nuclei or electrons in the silicon bulk. In particular the silicon's low nucleus mass ensures a good sensitivity to WIMPs with masses in the range 1-10 GeV, while its small band gap $(\sim 1.1 \mathrm{eV})$ provides sensitivity to hidden sector DM-electron interactions. The sensitivity to WIMP-nucleon interactions and to one possible hidden sector candidate is shown in Figure 1. To reach these results, DAMIC-M will require a radiogenic background rate of a fraction of d.r.u ${ }^{1}, 100$ times lower than DAMIC's one. Thus, an extensive campaign of innovation of the detector's technology and design is ongoing. Geant 4 simulations are being exploited to optimise the detector design, drive the material selection and handling and test background rejection techniques. In the following, the different investigated designs and the corresponding background levels will be described along with the background mitigation strategies which DAMIC-M is going to adopt.

\section{Experimental setup}

In the current design, DAMIC-M will employ 200 CCDs, each of them with a mass of about $5 \mathrm{~g}$. Every CCD will feature $6 \mathrm{k} \times 1.5 \mathrm{k}$ pixels over a $9 \mathrm{~cm} \times 2.25 \mathrm{~cm}$ area and a thickness of at least $0.675 \mathrm{~mm}$. CCDs are fabricated from n-type, high-resistivity silicon wafers and they feature a three-phase polysilicon gate structure with a buried p-channel [4]. Each CCD is fully depleted by applying a potential $\geq 40 \mathrm{~V}$ to a thin back-side contact.

The operation of a CCD applied to the DM search is based on the creation of electron-hole pairs due to nuclear/electronic recoils in the silicon bulk. Produced ionization charges are drifted by the applied electric field and diffuse transversely with a spatial variance proportional to the transit time (or depth of the interaction). This allows a 3D reconstruction of the energy deposits. The

\footnotetext{
${ }^{1} 1$ d.r.u (differential day units) corresponds to 1 event $/ \mathrm{day} / \mathrm{kg} / \mathrm{keV}$.
} 

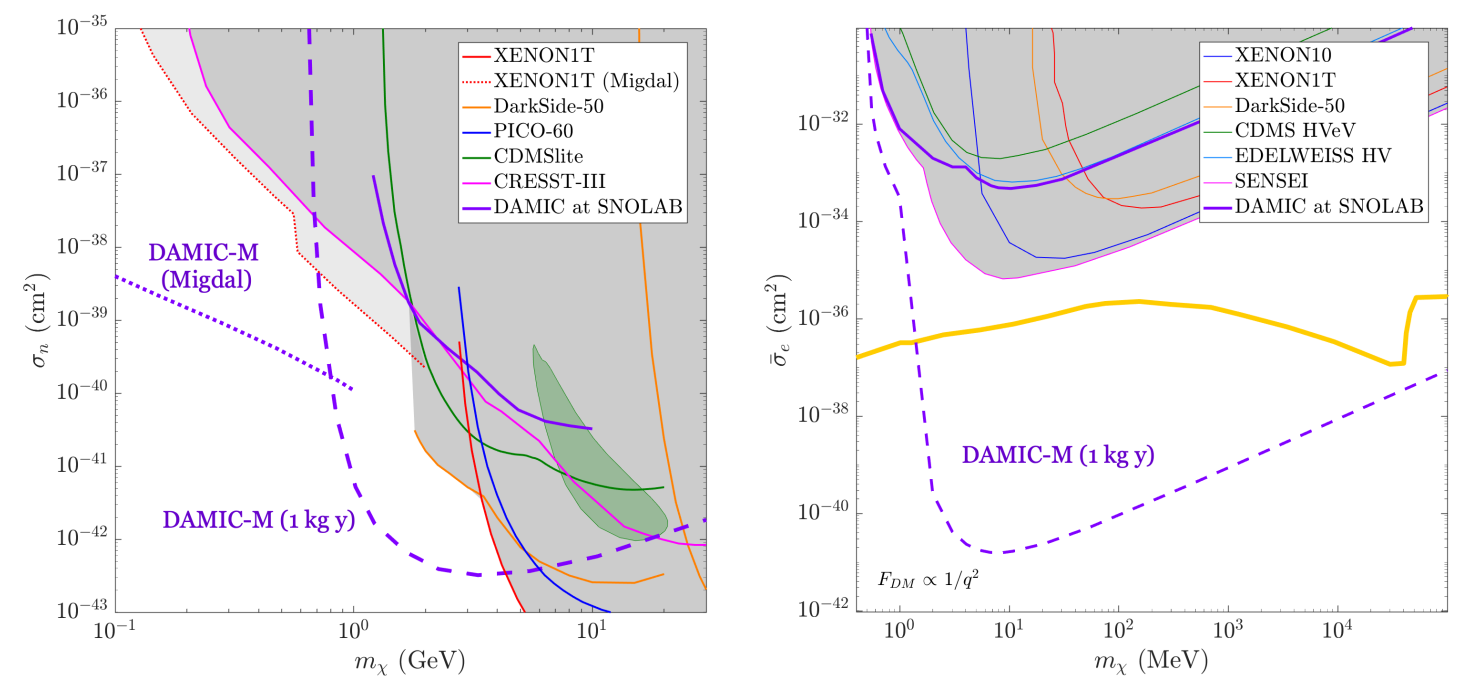

Figure 1: Examples of expected sensitivity of DAMIC-M [5] and exclusion limits compared to other dark matter searches and theoretical models. Left: exclusion limits for a WIMP-nucleon spin-independent scattering. The Midgal projection comes from [6]. Right: exclusion limit for a DM-electron interaction via a light dark photon mediator [2].

charges are collected and held at a polysilicon gate until read out. The new DAMIC-M CCDs will be equipped with "skipper" amplifiers which will perform repeated, non-destructive measurements of the charge in each pixel, in order to damp the readout noise (usually equal to $2 \mathrm{e}-\mathrm{rms} / \mathrm{pix}$ in conventional scientific CCDs) to a sub-electron level. Continuous readout will be used to minimize the accumulation of charge from leakage current in a pixel before it is read out. The CCD clocks and output node signal will travel on low background kapton cables or pico-coaxial cables.

The detector design is ongoing. The baseline scheme is shown in Figure 2 (left). A cryostat vessel will house the CCDs, packed in a box surrounded by infrared (IR) shields. Cooling will be provided by the use of liquid nitrogen, whose consumption will be optimized by a heat exchanger. Most of the components will be made of electro-formed (EF) copper, especially those nearest the CCDs, given their very low content of radioactive isotopes. This material requires a very long procedure to be grown and very high control of the background level to ensure its high purity. The farther volumes will instead be made of oxygen-free high thermal conductivity (OFHC) copper, widely used in cryogenics and characterized by high chemical purity. In the simple configuration similar to DAMIC's one, CCDs are disposed horizontally, on the top of each other (Figure 2, right (a)). Each CCD is held in a EF copper frame which ensures mechanical support for the CCD and cables and thermal contact. The background estimations presented in this proceedings are based on this option. Other configurations are under study as the one in Figure 2 (right (b)) with CCDs disposed vertically, hung on a copper support. A sandwich of electro-formed copper and ancient lead disks is placed on top of the CCD stack to shield from the background events from the above detector components. The simulations presented in the next sections were used to optimize the disks thickness. A polyethylene and ancient lead shields with a thickness of about $40 \mathrm{~cm}$ and $20 \mathrm{~cm}$ respectively will surround the detector to screen it from external neutrons and $\gamma$-rays. The whole 
detector design is still under development and not finalized.

\section{Background sources}

A major challenge for DAMIC-M is the control of the background to a level of a fraction of d.r.u. Main background sources are $\gamma$-rays, electrons and neutrons of radiogenic or cosmogenic origins, from materials surrounding the detector or in the silicon bulk.

Neutrons can be created by spallation of cosmic ray muons on nuclei in the materials around the experiment (prompt cosmogenic neutrons) or via radioactive processes. They can interact with the nuclei in the detector, inducing nuclear recoils. To reduce the background from prompt neutrons, direct dark matter experiments are hosted in underground laboratories and surrounded by a shield of high density polyethylene, which is an excellent neutron moderator. The radiogenic neutrons can instead be reduced by selecting materials with poor content of Uranium $\left({ }^{238} \mathrm{U}\right)$ and Thorium $\left({ }^{232} \mathrm{Th}\right)$.

Electrons and $\gamma$-rays are produced in the decays in the materials around and inside the experimental setup. $\gamma$-rays can produce electrons via photoelectric effect, Compton scattering and e+/e- pair production. Low energy electrons from Compton scattering are particularly critical at the energies of interest of DAMIC-M. Detailed measurements of Compton scattered electrons spectra in the bulk of skipper CCDs are currently being carried out [7]. The principal sources of electrons and $\gamma$-rays are the ${ }^{238} \mathrm{U}$ and ${ }^{232} \mathrm{Th}$ chains, the ${ }^{40} \mathrm{~K}$ and the cosmogenic isotopes. Cosmogenic isotopes are produced by spallation of cosmic rays in the detector components, like the copper or silicon ones. Particularly relevant is the case of the tritium $\left({ }^{3} \mathrm{H}\right)$ contamination, as shown by DAMIC results [8]. The ${ }^{3} \mathrm{H}$ production rate at energies below $5 \mathrm{keV}$ corresponds to an activity of roughly 0.002 decays/ ( keV kg day ) per day of sea-level exposure [9]. Moreover, traces of ${ }^{32} \mathrm{Si}$ and ${ }^{210} \mathrm{~Pb}$ can be found in the silicon bulk or surface. Their nuclei undergo sequential beta decays whose $\beta$ spectra extend to the low-energy region considered for the DM search. Given the unique spatial resolution of DAMIC-M, analysis techniques looking for spatial correlated events in the silicon bulk allow to identify these decay chains [10]. To reduce the radioactive isotopes contamination of the detector components, the DAMIC-M collaboration will employ high purity materials, strictly control the exposure time to cosmic rays by shielded transportation, underground storage and handling in a radon free ambient. Finally, the flux of $\gamma$-rays from the environment is suppressed by about $20 \mathrm{~cm}$ of archaeological lead which has low level of contamination of ${ }^{238} \mathrm{U}$ and ${ }^{232} \mathrm{Th}$. Another approach that DAMIC-M will use to lower surface background events, due to ${ }^{210} \mathrm{~Pb}$ contamination on CCD, is to remove about 10 microns from the backside surface of the silicon wafer. This technique would also result in the removal of the partial charge collection regions in the back of the CCD, which may lead to distortions in the low part of the energy spectrum [11]. Finally, further background rejection can be done at analysis level (e.g. selecting a fiducial volume to exclude surface background [4]).

The capability to control the backgrounds to the expected level will be demonstrated by the Low Background Chamber (LBC), DAMIC-M prototype which will use two 6k x 4k skipper CCDs and will be installed this year at the LSM laboratory. The LBC is meant to achieve a background level of few d.r.u. and it will provide a first validation of the background model down to single electron resolution. 


\section{Background simulations}

Detector simulations are performed to estimate the background level and drive the detector conception.

A Geant4 [12] code has been developed to simulate the physics processes undergone by a particle passing through the experimental setup. The code uses the Livermore physics list [13] provided with Geant 4 and includes neutron processes and radioactive decays. The Livermore lowenergy electromagnetic models are used to describe the interactions with matter of electrons and photons between $20 \mathrm{eV}$ and $100 \mathrm{GeV}$. The DAMIC-M detector design and the relative materials are implemented through a GDML (Geometry Description Markup Language) file, an applicationindependent geometry description format [12]. Production cuts are defined for $\mathrm{e}^{ \pm}, \gamma$ and protons to optimize the computing time without introducing energy spectrum deformations. The sensitive region and all the components close to the CCD have the lowest production cuts to ensure the maximum precision and minimum energy thresholds. The results reported here are obtained with Geant4 v.10.04 and by applying the following production cuts: $10^{-4} \mathrm{~mm}$ for the CCD, $3 \times 10^{-4}$ $\mathrm{mm}$ for the cables and copper components nearest to the CCD, $10^{-3} \mathrm{~mm}$ for the farthest copper components and $10^{-1} \mathrm{~mm}$ for ancient lead.

Once the energy deposits in the CCD are simulated, a code written in python (WADERS) is used to reproduce the detector response and provide the cluster reconstruction. The detector response includes processes like the division in pixels, dark current, electronic noise, pixel saturation and continuous readout. In the reconstruction all the contiguous charged pixels above the electronic noise threshold are collected in a cluster. Its total energy, 3D position and additional pattern parameters are calculated. For the purpose of the current studies the detector effects as diffusion, saturation, clustering threshold, dark current and readout noise are not included, being their effect negligible.

The DAMIC-M detector design is under continuous development. Each of the proposed designs is fully simulated to predict the corresponding background level and possibly propose further improvements. The simulated components for two example designs are shown in Figure 2.

The radioactive isotopes were uniformly simulated in the bulk of the detector components, choosing only the proper ones depending on the volume material. The following isotopes from the ${ }^{238} \mathrm{U}$ and ${ }^{232} \mathrm{Th}$ chains were considered: ${ }^{210} \mathrm{~Pb},{ }^{210} \mathrm{Bi},{ }^{212} \mathrm{~Pb},{ }^{212} \mathrm{Bi},{ }^{214} \mathrm{~Pb},{ }^{214} \mathrm{Bi},{ }^{234} \mathrm{Th},{ }^{234} \mathrm{~Pa}$, ${ }^{228} \mathrm{Ac},{ }^{208} \mathrm{Tl}$. The cosmogenic isotopes from copper activation were included: ${ }^{60} \mathrm{Co},{ }^{56} \mathrm{Co},{ }^{57} \mathrm{Co}$, ${ }^{58} \mathrm{Co},{ }^{54} \mathrm{Mn},{ }^{59} \mathrm{Fe}$. The ${ }^{40} \mathrm{~K}$ and the ${ }^{87} \mathrm{Rb}$ isotopes were also considered because traces of them can be found in epoxy and copper. The components of a radioactive chain were simulated separately. The background rate is obtained for each isotope scaling the cluster energy spectrum by a proper scale factor $\mathrm{f}$ :

$$
\mathrm{f}=\frac{\mathrm{n}_{\text {bins }} \cdot \mathrm{A}_{\text {iso }} \cdot \mathrm{m}_{\text {vol }}}{\Delta \mathrm{E} \cdot \mathrm{N}_{\text {decays }} \cdot \mathrm{M}_{\text {detector }}}
$$

where $n_{\text {bins }} / \Delta E$ is the bin width, $A_{\text {iso }}$ is the activity of the isotope in decays $/ \mathrm{kg} /$ day, $m_{\text {vol }}$ is the mass in $\mathrm{kg}$ of the volume of the detector in which the radioactive element is simulated, $\mathrm{M}_{\text {detector }}$ is the mass of the whole sensitive detector in $\mathrm{kg}$ and $\mathrm{N}_{\text {decays }}$ the number of simulated events. The used isotope activities were measured at SNOLAB for the DAMIC experiment or provided by the 


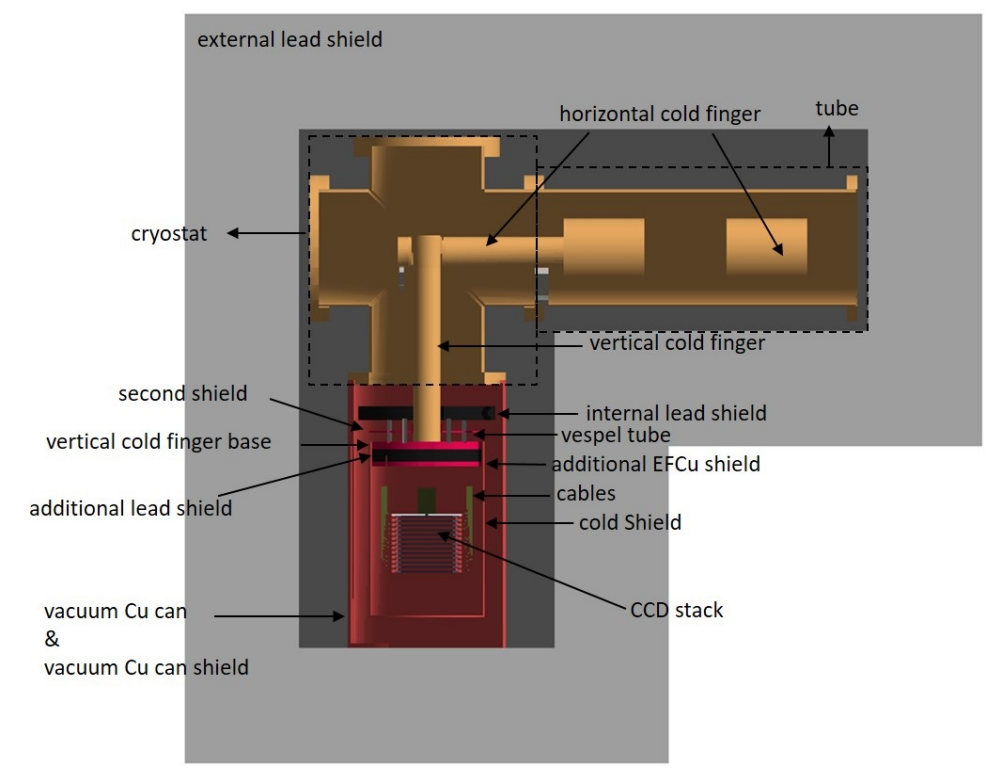

a)

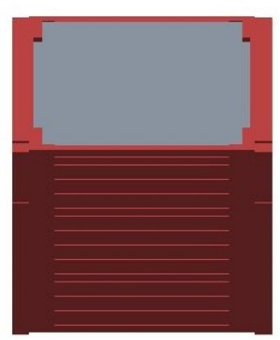

b)

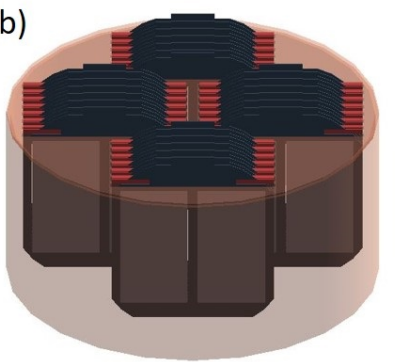

Figure 2: Left: latest simulated detector design. The electro-formed copper components are shown in red, while the OFHC copper ones in yellow. Right: zoom on two possible solutions for the CCD stack. a) Horizontal CCD stack. b) Vertical CCD stack. The gray parts are the CCDs, the red parts the copper holders.

material suppliers. An assay program is planned for all the detector materials. The used activities are reported in Table 1.

\begin{tabular}{|c|c|c|c|c|}
\hline Isotope & Copper & EF copper & Ancient Lead & Kapton cables (5 layers) \\
\hline${ }^{210} \mathrm{~Pb}$ & $2350 \pm 720$ & $<\mathbf{4 5 . 8}$ & $2850^{*}$ & $420^{\dagger}$ \\
\hline${ }^{232} \mathrm{Th}$ & $<3.5$ & $<0.0022$ & $0.2^{*}$ & $280 \pm 40$ \\
\hline${ }^{226} \mathrm{Ra}$ & $<11.2$ & $0.018^{\dagger}$ & $<\mathbf{2 . 0}$ & $420 \pm 490$ \\
\hline${ }^{238} \mathrm{U}$ & $<10.7$ & $<0.018$ & $<2.0$ & $5000 \pm 490$ \\
\hline${ }^{40} \mathrm{~K}$ & $<2.7$ & $2.7^{\dagger}$ & $<0.5$ & $2480 \pm 170$ \\
\hline${ }^{87} \mathrm{Rb}$ & $86.4^{\dagger}$ & $86.4^{\dagger}$ & - & $86.4^{\dagger}$ \\
\hline
\end{tabular}

Table 1: Activities in decays/day/kg per isotope and material used for simulations. The copper, kapton cables and ancient lead activities were measured by the DAMIC collaboration. The EF copper activities were provided by the material suppliers. The bold activities were decreased by a factor 10 , given the expected reasonable improvement in the material purity. $(\dagger)$ : in absence of measurements the ${ }^{87} \mathrm{Rb}$ activity was assumed to be $1 \mathrm{mBq} / \mathrm{kg}$, the activity of ${ }^{226} \mathrm{Ra}$ was assumed equal to that of ${ }^{238} \mathrm{U}$, the one of ${ }^{210} \mathrm{~Pb}$ to that of ${ }^{226} \mathrm{Ra}$ and the one of ${ }^{40} \mathrm{~K}$ to that of the copper. $(*)$ : measurement with unknown precision.

The activity of the cosmogenic isotopes is calculated based on the time of exposure to cosmic rays $\left(\mathrm{T}_{\exp }\right)$, the time spent underground before data collection ( $\left.\mathrm{T}_{\text {cool }}\right)$ and the running time of the experiment $\left(\mathrm{T}_{\text {run }}\right)$ :

$$
A=\text { Saturation } \cdot\left(1-\exp \left(-\lambda \mathrm{T}_{\exp }\right)\right) \cdot \exp \left(-\lambda \mathrm{T}_{\mathrm{cool}}\right) \cdot\left(1-\exp \left(-\lambda \mathrm{T}_{\text {run }}\right)\right) /\left(\lambda \mathrm{T}_{\text {run }}\right)
$$


where $\lambda=\log (2) / \mathrm{t}_{1 / 2}, \mathrm{t}_{1 / 2}$ is the half life of the isotope and Saturation is the cosmogenic production rate [14]. The exposure time, cooling time and run time of the copper components were fixed to 3 , 6 and 12 months respectively. The rate of background events was estimated by a fit to a constant in the energy interval between 2-7.5 keV. This energy range is chosen to exclude the silicon and copper fluorescence $\mathrm{K} \alpha$ emission peaks at $1.7 \mathrm{keV}$ and $8 \mathrm{keV}$ respectively. The total background estimation is shown in Figure 3 for the baseline design with CCDs stacked horizontally (Figure 2, left). The
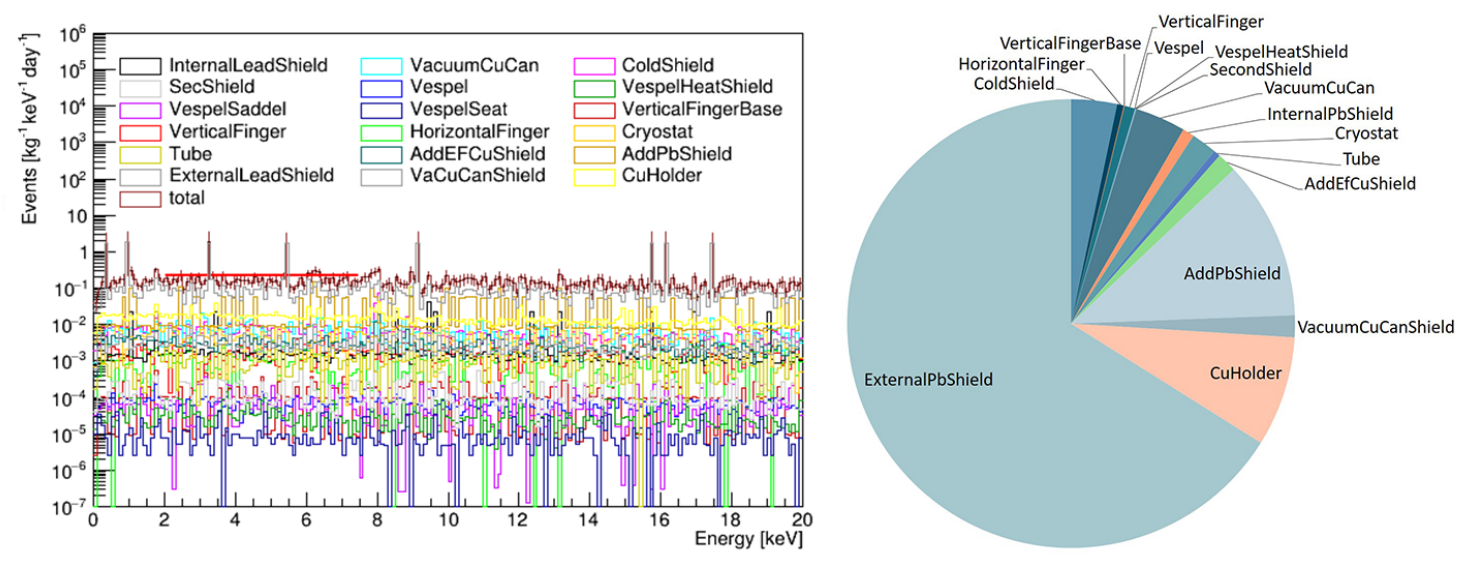

Figure 3: Left: total cluster energy spectrum and contribution from the individual components. The high peaks present in the total energy spectrum are due to Bi210 from the external lead shield. The red line is the result of a linear fit to a constant. Right: contribution to the background rate of each detector component. The total background rate is 0.2 d.r.u. Note that the used lead activities are upper limits.

total background rate is less than 0.2 decays $/ \mathrm{day} / \mathrm{keV} / \mathrm{kg}$, where $75 \%$ of it is due to the external lead shield. It is worth noticing that the background levels of the ancient lead components are based on weak activity upper limits. A dedicated assay will help to reduce this contributions. The result shown here doesn't take into account the cables and the cosmogenic activation of the electro-formed copper components. A study of the background rate for varying values of $\mathrm{T}_{\exp }$ and $\mathrm{T}_{\text {cool }}$ (with fixed $\mathrm{T}_{\exp }=1 \mathrm{y}$ ) has been performed helping to set the limits for the exposure to cosmic rays of the EF copper volumes. With $\mathrm{T}_{\exp }=10$ days and $\mathrm{T}_{\text {cool }} 6$ months, these components would contribute with 0.1 d.r.u. This result decreases by a factor 10 if we assume $T_{\exp }=1$ day and increases by a factor 3 if $\mathrm{T}_{\text {cool }}=0$. Finally, different options for the cables are under evaluation. The kapton cables (5-layers kapton cables) used by DAMIC at SNOLAB are discarded since they would produce a background of approximately 3 d.r.u, well beyond DAMIC-M's goal. Therefore two lower background options are being discussed: a 2-layers kapton cables and ultra low background pico-coaxial cables. In both cases, preliminary results show a reduction of the background rate of about a factor 10 with respect to the standard kapton cables. Precise background evaluations depend on the specific packaging and the CCD holder design.

\section{Conclusions}

A major objective of the DAMIC-M experiment is to lower the radiogenic background to a fraction of d.r.u. To this end, simulations are being performed to drive the detector design and the 
material selection and handling. The design is not yet finalized but current results show that the background level goal is within reach. The major contributors are the cables and the copper holder. The design with vertical CCDs reduces further the background by decreasing the amount of copper around the sensitive CCDs as well as the number of cables. In the background budget shown in Figure 3, the external lead shield looks as the dominant contributor. This result is however obtained with activities upper limits. Pivotal to better background evaluations are precise measurements of radiogenic isotope activities. Further studies are ongoing to perform fiducial cuts to reject part of the cable background events. The validity of simulations, especially at low energies, will be tested with the LBC which is going to be installed this year.

The authors are supported by the CNRS-University of Chicago fellowship program. This work was done within the DAMIC-M Collaboration.

\section{References}

[1] N. Aghanim et al. [Planck], Astron. Astrophys. 641 (2020), A6 [arXiv: 1807.06209].

[2] R. Essig, J. Mardon and T. Volansky, Phys. Rev. D 85 (2012), 076007 [arXiv: 1108 . 5383].

[3] J. Tiffenberg et al. [SENSEI], Phys. Rev. Lett. 119 (2017) no.13, 131802 [arXiv: 1706.00028].

[4] A. Aguilar-Arevalo et al. [DAMIC], Phys. Rev. D 94 (2016) no.8, 082006 [arXiv: 1607.07410].

[5] P. Privitera for the DAMIC-M collaboration, in proceedings of TAUP Conference (2019).

[6] S. Knapen, J. Kozaczuk and T. Lin, [arXiv:2011.09496].

[7] J. Cuevas-Zepeda, D. Norcini, P. Privitera, APS April Meeting (2021).

[8] A. Aguilar-Arevalo et al. [DAMIC], Phys. Rev. Lett. 125 (2020), 241803 [arXiv:2007.15622].

[9] R. Saldanha, R. Thomas, R. H. M. Tsang, A. E. Chavarria, R. Bunker, J. L. Burnett, S. R. Elliott, A. Matalon, P. Mitra and A. Piers, et al.Phys. Rev. D 102 (2020) no.10, 102006 [arXiv: 2007.10584].

[10] A. Aguilar-Arevalo et al. [DAMIC], [arXiv:2011.12922].

[11] G. Fernandez-Moroni, K. Andersson, A. Botti, J. Estrada, D. Rodrigues and J. Tiffenberg, [arXiv: 2007.04201].

[12] S. Agostinelli et al. [GEANT4], Nucl. Instrum. Meth. A 506 (2003), 250-303.

[13] J. Allison, J. Apostolakis, S. B. Lee, K. Amako, S. Chauvie, A. Mantero, J. I. Shin, T. Toshito, P. R. Truscott and T. Yamashita, et al. Nucl. Instrum. Meth. A 835 (2016), 186-225.

[14] M. Laubenstein, G. Heusser, Appl Radiat Isot. 67 (2009), 750-754. 